\title{
Síndrome de Ogilvie o pseudobstrucción aguda del colon: una causa rara de abdomen agudo, de elevada mortalidad y asociada a patologías frecuentes en un Servicio de Medicina Interna
}

\author{
C. MONTERO LEAL, R. M. HORMEÑO BERMEJO, E. GONZÁLEZ MORENO, \\ B. GORDILLO MORERA
}

Servicio de Medicina Interna. Hospital Perpetuo Socorro. Badajoz

THE ACUTE PSEUDOBSTRUCTION OF THE COLON OR OGILVIE'S SYNDROME

\begin{abstract}
RESUMEN
La pseudobstrucción aguda del colon o síndrome de Ogilvie es una entidad clínica rara de curso agudo o subagudo que se asocia a diferentes patologías clínicas o quirúrgicas, y cuya etiopatogenia sigue siendo desconocida. El tratamiento se inicia con medidas generales y si estas no son efectivas, con la descompresión quirúrgica del colón para evitar la complicación más grave y frecuente que es la perforación cecal. Presentamos el caso de una dilatación aguda e idiopática del colón en un varón adulto que ingresó por un episodio de insuficiencia respiratoria aguda, y que se resolvió con tratamiento médico conservador.
\end{abstract}

PALABRAS CLAVE: Colon. Pseudobstrución de colon. Dilatación. Abdomen agudo. Síndrome de Ogilvie.

\begin{abstract}
The acute pseudobstruction of the colon or Ogilvie's syndrome is a infrecuent pathology of acute or subacute evolution, that is associated to different clinical or surgical pathologies, and whose pathophysilogy continues being not known. The treatment begins with general measures and if these are not effective, with the surgical decompression of colon to avoid the most serious complication and it frequents that it is the perforation of the ceacum. We present a case of an acute and idiopathic dilatation of colon in an adult man who entered by an episode of acute respiratory insufficiency, and that was solved with preservative medical treatment.
\end{abstract}

KEY WORDS: Colon. Pseudo-obstruction. Dilatation. Acute abdomen. Ogilvie's syndrome.

Montero Leal C, Hormeño Bermejo RM, González Moreno E, Gordillo Morera B. Síndrome de Ogilvie o pseudobstrucción aguda del colon: una causa rara de abdomen agudo, de elevada mortalidad y asociada a patologías frecuentes en un Servicio de Medicina Interna. An Med Interna (Madrid) 2006; 23: 435-437.

\section{INTRODUCCIÓN}

El síndrome de Ogilvie o pseudobstrución aguda del colon se caracteriza por una dilatación masiva del colon y clínica sugestiva de obstrucción intestinal mecánica sin causa orgánica que justifique dicha dilatación. En la actualidad se considera una entidad infrecuente y se asocia a patologías médicas o quirúrgicas, principalmente a traumatismos, cirugía, procedimientos obstétricos o enfermedad neurológica. El tratamiento es la descompresión colónica precoz mediante medidas generales y fármacos para disminuir la distensión del colon; si estas no son efectivas se debe recurrir a técnicas endoscópicas y cirugía. La mortalidad intrahospitalaria es de un $30 \%$ y aumenta cuanto mayor es el diámetro cecal afecto ya que mayor es el índice de perforación, por lo que es importante para el clínico el reconocimiento, diagnóstico y tratamiento de este síndrome.

\section{CASO APORTADO}

Paciente varón de 82 años de edad con antecedentes personales de EPOC, Cor-pulmonale, hipertensión pulmonar severa, hipercolesterolemia, hiperuricemia e hipertensión arterial. Seguía tratamiento oral ambulatorio con torasemida, enalapril y pentoxifilina, así como broncodilatadores por vía inhalatoria (agonista $\mathrm{B}_{2}$, bromuro de ipatropio y corticoides). Ingresó en nuestro hospital por aumento progresivo de su disnea habitual hasta hacerse de reposo con ortopnea y episodios de DPN, acompañado de tos, expectoración amarillenta y edemas en miembros inferiores, sin fiebre ni vómitos y hábito intestinal regular con tendencia al estreñimiento.En la exploración, el paciente estaba consciente, vigil, colaborador, afebril, bien hidratado y prefundido, taquipneico (FR 26 respiraciones/min), y normotenso. La auscultación pulmonar revelaba roncus y sibilancias diseminadas en ambos hemitórax y algunos crepitantes bibasales. La auscultación cardiaca era rítmica a unos 70 latidos/min. La exploración abdominal mostraba una gran distensión del hemiabdomen superior con gran timpanismo pero sin puntos dolorosos ni defensa, sin signos de irrita-

Trabajo aceptado: 21 de marzo de 2006 
ción peritoneal y con ruidos hidroaéreos presentes pero con algunos metálicos. En el tacto rectal el dedil era limpio, no dolorosos y no se tocaban masas. La analítica mostraba una hemoglobina de 14,8 g/dl; hematocrito 42,4\%; volumen corpuscular medio (VCM): 93,9 fl; leucocitos: $18,80 \times 10^{9} / 1(17.000 \mathrm{NS}, 800 \mathrm{~L}, 300 \mathrm{M})$; actividad de protrombina 110,6\%.TTPA 30,9 segundos; (normal hasta $275 \mathrm{ng} / \mathrm{ml}$ ); glucosa $175 \mathrm{mg} / \mathrm{dl}$; urea $150 \mathrm{mg} / \mathrm{dl}$; creatinina $1,54 \mathrm{mg} / \mathrm{dl}$, ácido úrico 10,3 mg/dl, LDH 848 UI/I, GPT 56 UI/I. La gasometría arterial reflejaba: ph 7,45/pCO2 32,5/pO2 59,5/Bi 22,4/Sat O2 94,3\%. Los estudios radiológicos que se realizaron revelaban los siguientes resultados: $\mathrm{La}$ radiografía de torax : Signos de EPOC sin otros datos patológicos. La radiografía simple de abdomen en decúbito (Fig. 1) y bipedestación: Gran dilatación de asas del intestino grueso (más de 6-7 centímetros de diámetro) sin niveles hidroaéreos sugestivos de obstrucción mecánica. La TAC de abdomen y enema opaco con contraste hidrosoluble rectal mostraban un transverso, colon ascendente y ciego dilatados pero descartaban causa obstructiva mecánica.

Se inició tratamiento con broncodilatadores, tanda corta de corticoides, amoxicilina clavulánico, diuréticos y oxigenoterapia, y se suspendió tratamiento con verapamil. Igualmente se instauró tratamiento con sonda rectal, enemas de limpieza, dieta absoluta, hidratación intravenosa con reposición electrolítica. El paciente mejoró progresivamente estando al alta vigil, orientado, colaborador, con buen estado general, eupneico en reposo, tolerando bien la dieta oral y presentando deposiciones normales y de forma espontánea.

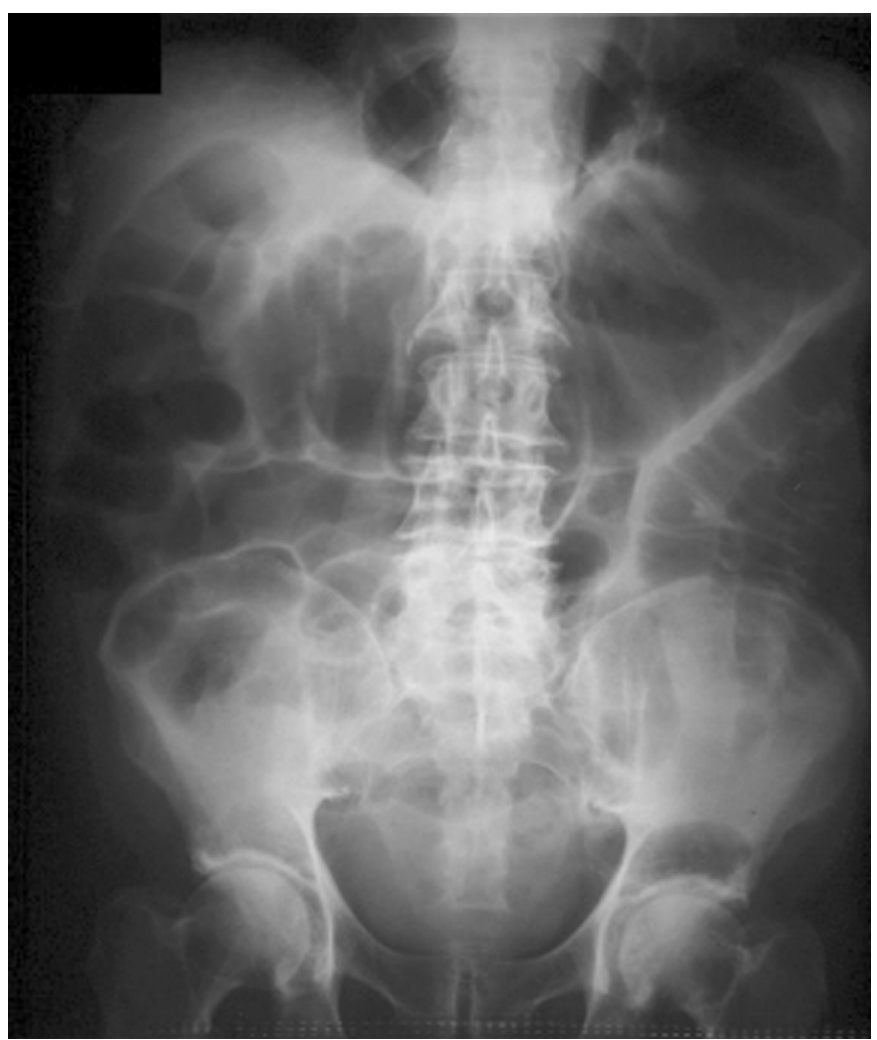

Fig. 1.

\section{DISCUSIÓN}

El síndrome de Ogilvie descrito por primera vez en 1948 por sir Heneage Olgivie (1) es una dilatación idiopática y progresiva del colón. Se trata de una entidad poco frecuente y que predomina en varones de edad avanzada (2). La etiolo- gía sigue siendo desconocida habiéndose descrito asociado a otras patologías como sepsis, quemaduras, traumatismos, cirugía pelviana y abdominal, insuficiencia a respiratoria o enfermedad neurológica (3-5) (Tabla I). La causa que origina el cuadro no está clara; Ogilvie inicialmente postuló que la pared del colón y el recto pudiera relajarse en respuesta a estímulos fisiológicos y farmacológicos, produciéndose un desequilibrio autonómico del control intrínseco como base del cuadro. Esta hipótesis ha sido confirmada al obtenerse un alivio de los síntomas por medio de un bloqueo adrenérgico seguido por la estimulación colinérgica o por neostigmina sola $(6,9)$. La clínica y la radiología son muy similares al de una obstrucción mecánica del colón. Los síntomas tienen un comienzo agudo y consisten en meteorismo, nauseas, vómitos, estreñimiento o diarrea. Al examen físico el abdomen se presenta distendido, timpánico, con escasos o nulos ruidos intestinales y puede existir defensa en fosa ilíaca derecha $(3,9)$. La principal dilatación ocurre normalmente en el colón derecho sobre todo en el ciego. El cuadro suele evolucionar de forma progresiva a la dilatación, isquemia de la pared, perforación y muerte. Si el ciego se dilata más de 910 centímetros (medido en la radiografía de abdomen), el riesgo de perforación es inminente $(7,8)$. El diagnóstico de este síndrome es difícil ya que clínicamente es imposible diferenciarlo de un íleo mecánico y en algunas ocasiones la exclusión de una causa orgánica requiere laparotomía exploradora. Este se establece en base a la historia clínica, la exploración física y el estudio radiológico (radiografía simple de abdomen) (4). La mortalidad es elevada siendo de mal pronóstico la edad avanzada, enfermedades asociadas, el tiempo de evolución, el diámetro del ciego, la necrosis y la perforación $(5,8,9)$. El tratamiento está en función del estado del paciente y la severidad de los síntomas. Si el ciego tiene menos de 12 centímetros de diámetro, debe iniciarse tratamiento conservador (ayuno, sonda rectal, enemas de limpieza, reposición y corrección hidroelectrolítica y fármacos), pero si existe un incremento progresivo del colón o el cuadro clínico se agrava, se debe realizar una descompresión colónica precoz (colonoscopia o rectoscopia) y si esta falla se recurrirá al tratamiento quirúrgico (cecostomía o hemicolectomía derecha). En todos los casos se deben administrar

\section{TABLA I}

SITUACIONES DE RIESGO DEL SÍNDROME DE OGILVIE
1. Ancianos
2. Enfermedades vasculares, cardiacas, neurológicas, respiratorias, hematológicas.
3. Tóxicos.
4. Infecciones graves
5. Traumatismos
6. Alcoholismo.
7. Cirugía pelviana y abdominal.
8. Yatrogenia.
9. Factores metabólicos.
10. Enfermedad diverticular del colon.
11. Fármacos: calcioantagonistas, espasmolíticos, neuro- lépticos, antidepresivos tricíclicos, opioides, corticoi- des, antihistaminicos $\mathrm{H} 2$.
12. Diabetes, hipotiroidismo 
líquidos intravenosos y realizar las correcciones electrolíticas necesarias $(3,4,7)$. En cuanto a medidas farmacológicas, estudios recientes hacen referencia a la importancia de drogas inhibidoras de la acetilcolinesterasa como la neostigmina en el tratamiento de este síndrome. La neostigmina, estimula el sistema nervioso autónomo parasimpático restableciendo el peristaltismo. Se utiliza en bolo intravenoso único a la dosis de 2-2,5 mg. Otros fármacos como los agonistas de los receptores de la 5-hidroxitriptamina están aún en estudio $(7,9,10)$. En nuestro caso tras realizarse el estudio radiológico que descarta causa obstructiva, tratamiento de la insuficiencia y sobreinfección respiratoria, así como las medidas de ayuno, sonda rectal, enemas de limpieza y reposición hídrica y de electrolíticos, el paciente mejoró de forma lenta y progresiva hasta recibir el alta hospitalaria.

Dada la elevada tasa de mortalidad de este síndrome y su mayor incidencia en pacientes de avanzada edad (pacientes cada vez más frecuentes en los servicios de medicina interna), es necesario tenerlo presente para realizar un diagnóstico y tratamiento precoz.

\section{Bibliografía}

1. Nani G, Garbini A, Luchetti P, Ronconi P, Castanegto M. Ogilvie,s syndrome (acute colonic pseudo-obstrucion): review of literature (October 1948 to march 1980) and report of four additional cases. Dis Colon Rectum 1982; 25: 157-66.

2. Rondeau M, Weber JC, Nodot I, Storck D. Acute colonic pseudo-obstruction in internal medicine: etiology and prognosis, report of a retrospective study. Rev Med Interne 2001; 22: 536-4.

3. Cross JM, Marvin RG: Acute Colonic Pseudo-Obstruction in a Patient with a Significant Closed Head Injuri. The Internet Journal of Emergency and Intensive Care Medicine 2000; 4 (1).

4. Campillos Páez MT, Pardo Moreno G, Campillos Páez A. Síndrome de Ogilvie: a propósito de un caso. Medifam 2002; 12: 593-596.

5. Vega Basulto SD, Mosquera Betancourt G, Varela Hernández A. Ogilvie,s Síndrome. A report of 3 cases. Neurocirugia 2002; 13: 229-32.

6. Trevisani GT, Hyman NH, Church JM. Neostigmine: safe and effective

treatment of acute colonic pseudo-obstruction. Dis Colon Rectum 2000; 43: 1454 .

7. De Giorgio R, Barabar G, Stanghellini V, Tonini M, Vasina V, Ciola J, et al. Review article: the pharmacological treatment of acute colon pseudos-obstruction. Aliment Pharmacol Ther 2001; 15: 1717-27.

8. Árbol Linde F, Marcos Sánchez F, Contreras Rosino C, Sarmiento EJ, López Onega P. Perforación de ciego de curso fatal en la evolución de un síndrome de Ogilvie. An Med Interna (Madrid) 1997; 14: 75-76.

9. Delgado Aros S, Camilleri, M. Clinical managment of acute colonic psudo-osbtruction in patient: a systematic review of literature. Gastroenterol Hepatol 2003; 26: 646-55.

10. Turégano Fuente F, Muñoz Jiménez F, del Valle Hernández E, Pérez Díaz D, Calvo Serrano M, De Tomás J, et al. Early resolution of Olgivie,s síndrome with intravenous neostigmine: a simple, effective treatment. Dis Colon Rectum 1997; 40: 1353-57. 